\title{
The Transmittances of Thin Polymer Films and Their Suitability as a Supportive Substrate for a Soft X-ray Solar Filter
}

\author{
Memorie Williams \\ Brigham Young University - Provo \\ Evan Hansen \\ Brigham Young University - Provo \\ A. Reyes-Mena \\ Brigham Young University - Provo \\ David D. Allred \\ pllifor@piys.afly additional works at: https://scholarsarchive.byu.edu/facpub \\ Part of the Astrophysics and Astronomy Commons, and the Physics Commons
}

\section{Original Publication Citation}

Memorie Williams, Evan Hansen, A. Reyes-Mena, and David D. Allred, "The Transmittance of Thin Polymer Films and their Suitability as a Support Substrate for a Soft X-ray Telescope," EUV, X Ray, and Gamma Ray Instrumentation for Astronomy II, Proceedings of SPIE 1549, 147154 (1991). http://spiedigitallibrary.org/proceedings/resource/2/psisdg/1549/1/ 147_1?isAuthorized=no http://dx.doi.org/1.1117/12.48335

\section{BYU ScholarsArchive Citation}

Williams, Memorie; Hansen, Evan; Reyes-Mena, A.; and Allred, David D., "The Transmittances of Thin Polymer Films and Their Suitability as a Supportive Substrate for a Soft X-ray Solar Filter" (1991). Faculty Publications. 1353.

https://scholarsarchive.byu.edu/facpub/1353

This Peer-Reviewed Article is brought to you for free and open access by BYU ScholarsArchive. It has been accepted for inclusion in Faculty Publications by an authorized administrator of BYU ScholarsArchive. For more information, please contact ellen_amatangelo@byu.edu. 


\title{
THE TRANSMITTANCES OF THIN POLYMER FILMS AND THEIR SUITABILITY AS A SUPPORTIVE SUBSTRATE FOR A SOFT X-RAY SOLAR FILTER
}

\author{
Memorie Williams, Evan Hansen, A. Reyes-Mena, David D. Allred \\ Brigham Young University, Dept. of Physics, Provo, Utah, 84602
}

\begin{abstract}
This paper discusses soft $\mathrm{x}$-ray filter designs for the Brigham Young University "Goldhelox Project". Three polymers intended for use as a supportive substrate for a soft x-ray solar filter, having a passband centered at 171 angstroms are examined. The use of polymer substrates is examined because of vibrational and mechanical stresses associated with the shuttle launch, preventing the use of a free standing filter, and because of Goldhelox's special need to locate the filter near the imaging plane. The uniform consistency of a polymer support will prevent any imaging of the filter support structure, as would occur if a traditional mesh support was used. The polymer substrates investigated are: AP-1, Formvar, and polypropylene. Their transmissive characteristics of the polymers are examined along with the feasibility of their use. Transmission as a function of energy for each polymer is given over an energy range of 10 to $180 \mathrm{eV}$.
\end{abstract}

\section{INTRODUCTION}

The Goldhelox Project is a student-run research organization that is building a nearnormal incidence soft $x$-ray robotic telescope. Goldhelox is working in conjunction with the NASA Get-Away-Special (GAS) program, which will allow us to fly our telescope above the atmosphere on one of its shuttles and photograph the solar corona at a wavelength band of 171 . 181 angstroms. The target date for completion of the Goldhelox Project is the fall of 1991 . After completion our telescope will be scheduled to fly on the next compatible shuttle mission.

Thin film filters play an important role in soft $x$-ray astronomy. To image heavenly bodies in EUV and soft $x$-ray wavelengths radiation of all other wavelengths which reach the detector and to which the detector is sensitive must reject or attenuate without impairing soft $\mathrm{x}$ ray observations. Since our detector is insensitive to wavelengths longer than 2000 angstroms, the UV, visible, and IR spectrums will be attenuated solely to avoid overheating the detector.

The thin film filters used in $\mathrm{x}$-ray astronomy can be separated into two general categories based upon method of structural support: mesh supported and non-mesh supported. Because soft $\mathrm{x}$-rays do not penetrate, soft $\mathrm{x}$-ray filters are extremely thin and delicate and their lifetime is considerably shortened if used without support. Fine metal screen meshes are the most common method of providing support currently in use, and are especially useful for supporting large area fiiters. Non-mesh supported filters usually make use of the strength of a thin plastic or polymer for structural integrity and can be described as polymer supported. Both means of filter support have advantages and disadvantages. 
Meshes make excellent filter supports because they are sturdy and transmit incident $x$ rays well. Strong, supportive meshes capable of transmitting more than $90 \%$ of incident $x$-rays are commonly used in this application. However, meshes do produce some undesirable optical effects that have been documented by Hunter ${ }^{1}$. A mesh placed near to the focal plane results in a shadow cast upon the image where the wires block incident rays. Meshes placed farther from the focal plane result in diffraction effects and loss of resolution that degrade image quality.

Polymer supported filters are useful because of their strength, their transmission characteristics, and their absence of the detrimental optical effects caused by meshes. However, polymers have limited usefulness in EUV and soft $\mathrm{x}$-ray applications, as frequently the structurally necessary thickness cannot transmit a satisfactory percentage of the incident wavelengths of interest. Since the design of the Goldhelox telescope requires that the filter be placed very near the image plane, the use of a mesh support would be detrimental to the image produced by the telescope. For this reason, we have elected to investigate polymeric films as a possible support for the filtering components for the Goldhelox telescope. This paper presents the results of transmission measurements of thin films of Advanced Polymer 1 (AP-1), polypropylene, and Formvar.

The polymer AP-1 is currently manufactured by MOXTEK $^{2}$ as part of a ultra-high transmission $x$-ray window. It was selected by reason of it's availability, ease in manufacturing, and current application as a filter support. The second polymer selected as a possibility for a suitable filter substrate is polypropylene. Films of polypropylene were investigated by Spiller ${ }^{3}$ as a support, but were rejected by him in favor of Parylene $\mathrm{N}$, which has higher heat stability. However, due to its low density and lack of absorbing elements other than carbon, polypropylene is expected to be very transmissive. This expectation is born out by the calculations of Spivack ${ }^{4}$. The final material, Formvar, has been used extensively as an $\mathrm{x}$-ray window material. Its transmission has been investigated by Henke ${ }^{5}$ but outside the region of our investigation. It is slightly denser than Lexan, polypropylene or parylene. The elements from which Formvar is composed exhibit no absorption edges near $171 \AA$. It is of interest because it can be made into very thin films and because it is easy to manufacture.

\section{EXPERIMENTAL SETUP}

The experiment was performed at the ALADDIN synchrotron facility in Stoughton, Wisconsin using the 6-meter toroidal grating monochromator (TGM) soft $x$-ray beamline as the radiation source, and using one of the experimental chambers owned by Vanderbuilt University and operated by Dr. Norman Tolk's research group. The toroidal grating monochromator utilizes three different gratings to define the energy passing through the beamline, a low energy grating (LEG), a medium energy grating (MEG), and a high energy grating (HEG). The useful energy range of the TGM is about $10 \mathrm{eV}$ to $180 \mathrm{eV}$. The reflectivity of these three gratings is not constant throughout their useful energies and they may have other peculiarities. However, due to the nature of our setup their properties should not have affected our experiment.

Fig. I shows the experimental setup. Two fine $90 \%$ transmitting nickel meshes were used, one being placed between the source and sample, the other directly behind the sample. 
The photoelectric currents generated on the two meshes by the synchrotron radiation were read simultaneously by the computer. These readings, taken every $0.1 \mathrm{eV}$ throughout the useful energy range, were then normalized to prevent time variations and other characteristics of the radiation source from affecting our measurements. The data was taken in read cycles 300 data points wide, with a time delay of about 5 minutes between each cycle required tosave the data and reset the computer. The currents on the two meshes were also measured without a sample between them to determine

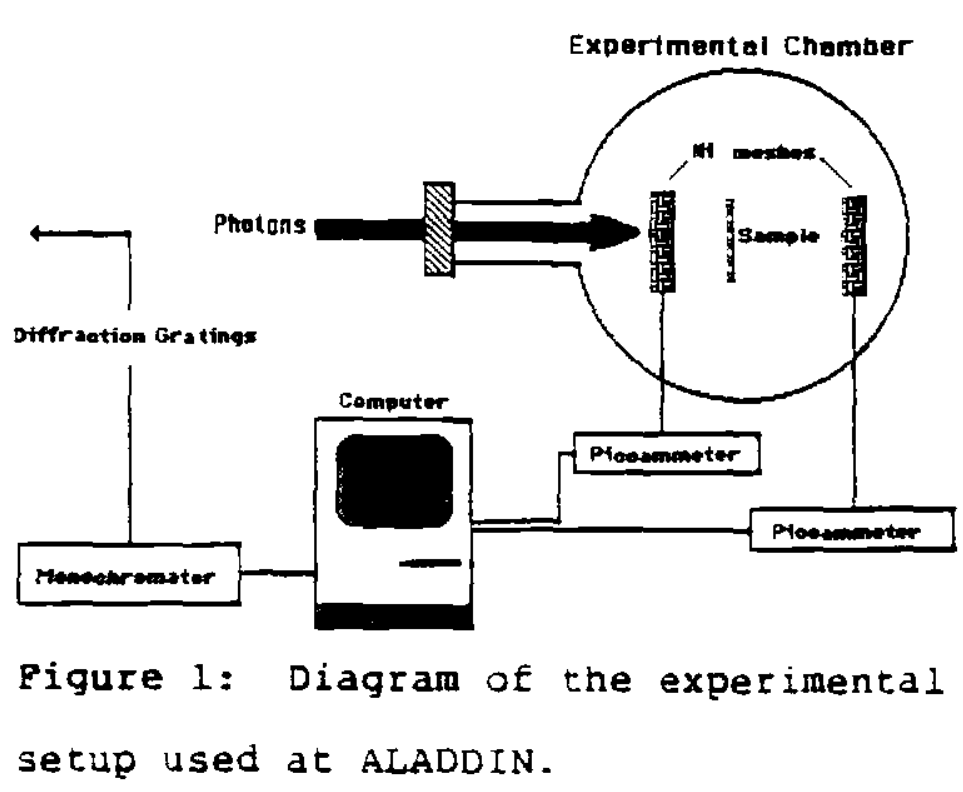
the relative detection efficiencies of each mesh. Furthermore, the size of the spacing of the wires of the mesh used for the detectors is small in comparison the size of the beam, and the position of the meshes was not changed at any point during the experiment. The three polymer films used for this study were prepared at BYU. Their effective thicknesses were determined from their weight and were found to be $2900 \AA$ for polypropylene, $1080 \AA$ for AP1, and 3600 $\dot{A}$ for Formvar.

\section{RESULTS AND DISCUSSION}

During the time allotted to us at ALADDIN, the transmission of three free standing polymer films was measured. The results presented in this paper are a preliminary study of their transmissive properties.

The fractional transmission of polypropylene is shown over the entire useful energy range of the beamline in fig 2 . The solid curve is a computed theoretical prediction of the transmission based upon the Henke tables. The overall shape of the measured data does follow the theoretical predictions quite well. The curves start with moderate transmission at the lowest energy, about $10 \%$ at $10 \mathrm{eV}$, falling to $5 \%$ at $20 \mathrm{eV}$ and rising gradually to a maximum transmission of $80 \%$ at $140 \mathrm{eV}$. The discontinuous jump in transmission that occurred at $35 \mathrm{eV}$ occurs at the same energy as the change from the low energy grating to the medium energy grating of the TGM. This jump may be due to the change in the experimental gratings, but we have not been able to determine a mechanism to account for this. The next discontinuity occurs at about $57 \mathrm{eV}$. This and the feature at $65 \mathrm{eV}$ do not appear to correspond to any instrumental artifacts. The shift from the medium energy grating to the high energy grating occurs at $80 \mathrm{eV}$. Here the curve continues smoothly upwards as expected, albeit with considerably more scattering in the data, which may be due to the low reflective efficiency of the HEG. As was discussed, the data was collected in increments. The discontinuities at 110 and $140 \mathrm{eV}$ occur at these increments, but none of the others correspond with any similar instrumental or procedural idiosyncracies. Although the data is merely preliminary, the discontinuities observed are 


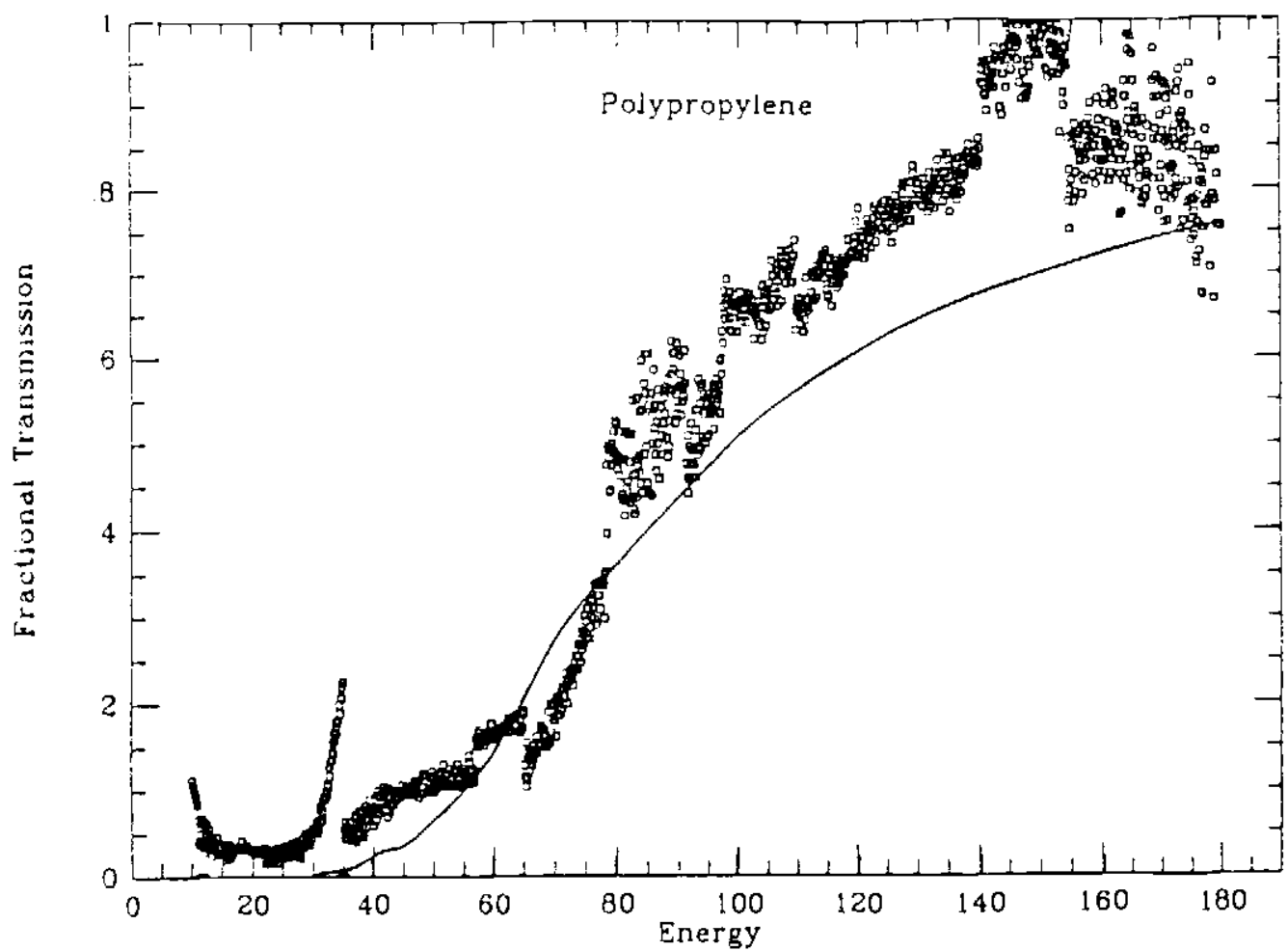

Figure 2: The fractional transmission of polypropylene plotted with respect to energy. The solid curve is the theoretical transmission based upon the absorption coefficients of its composition elements as found in the Henke tables.

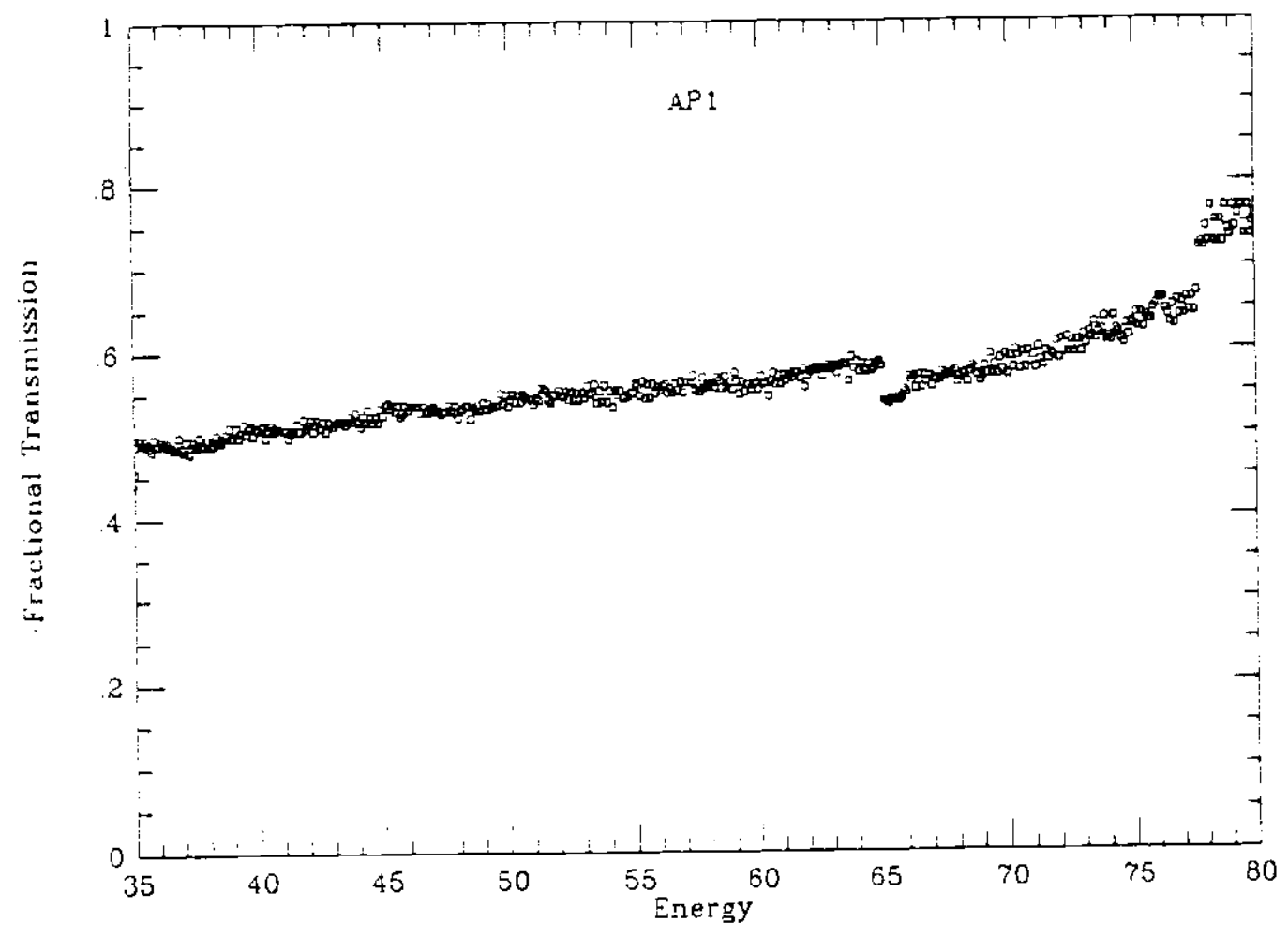

Figure 3: The fractional transmission of AP-1 plotted with respect to energy. 


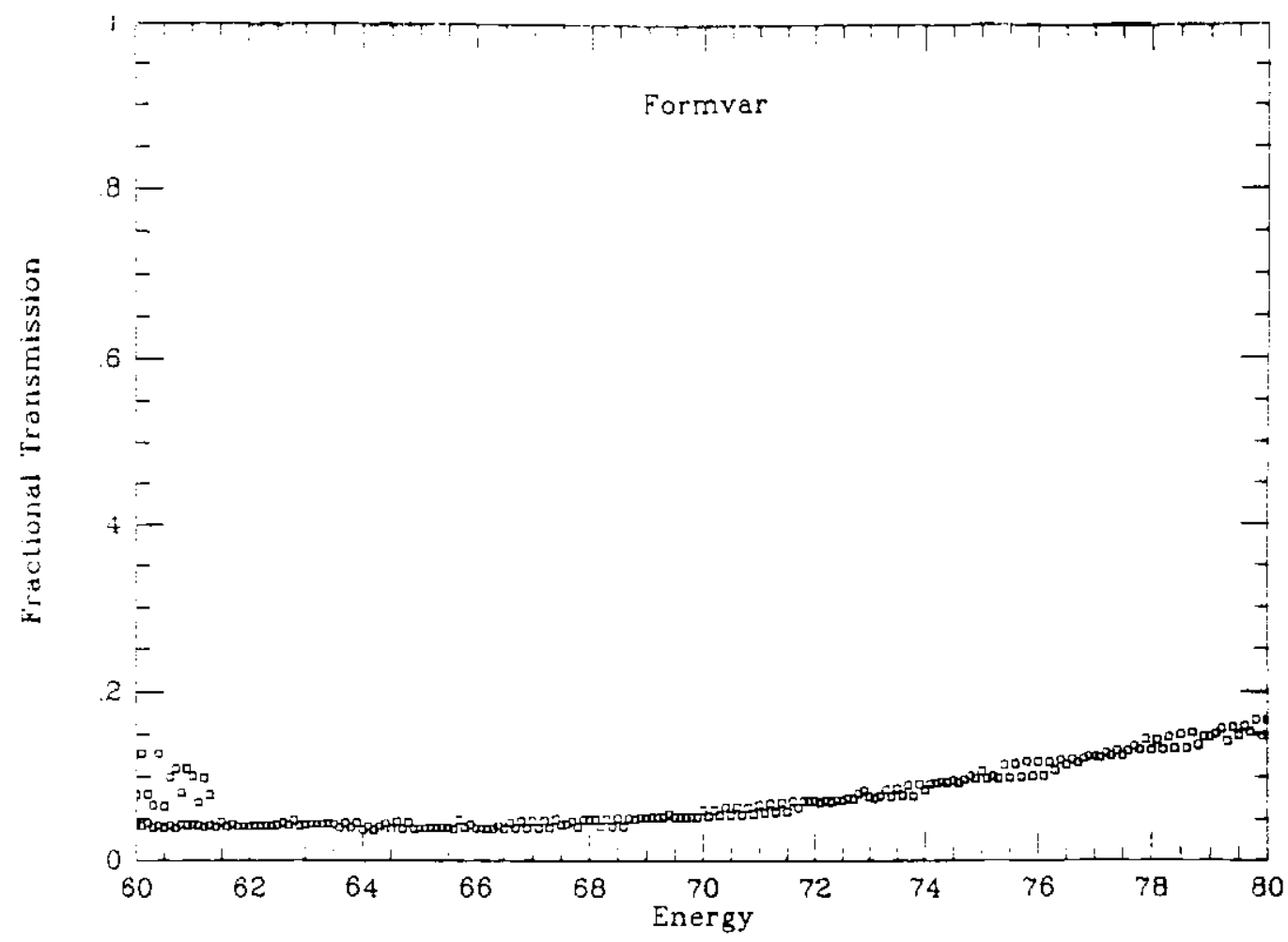

Figure 4: The fractional transmission of Formvar plotted with respect to energy.

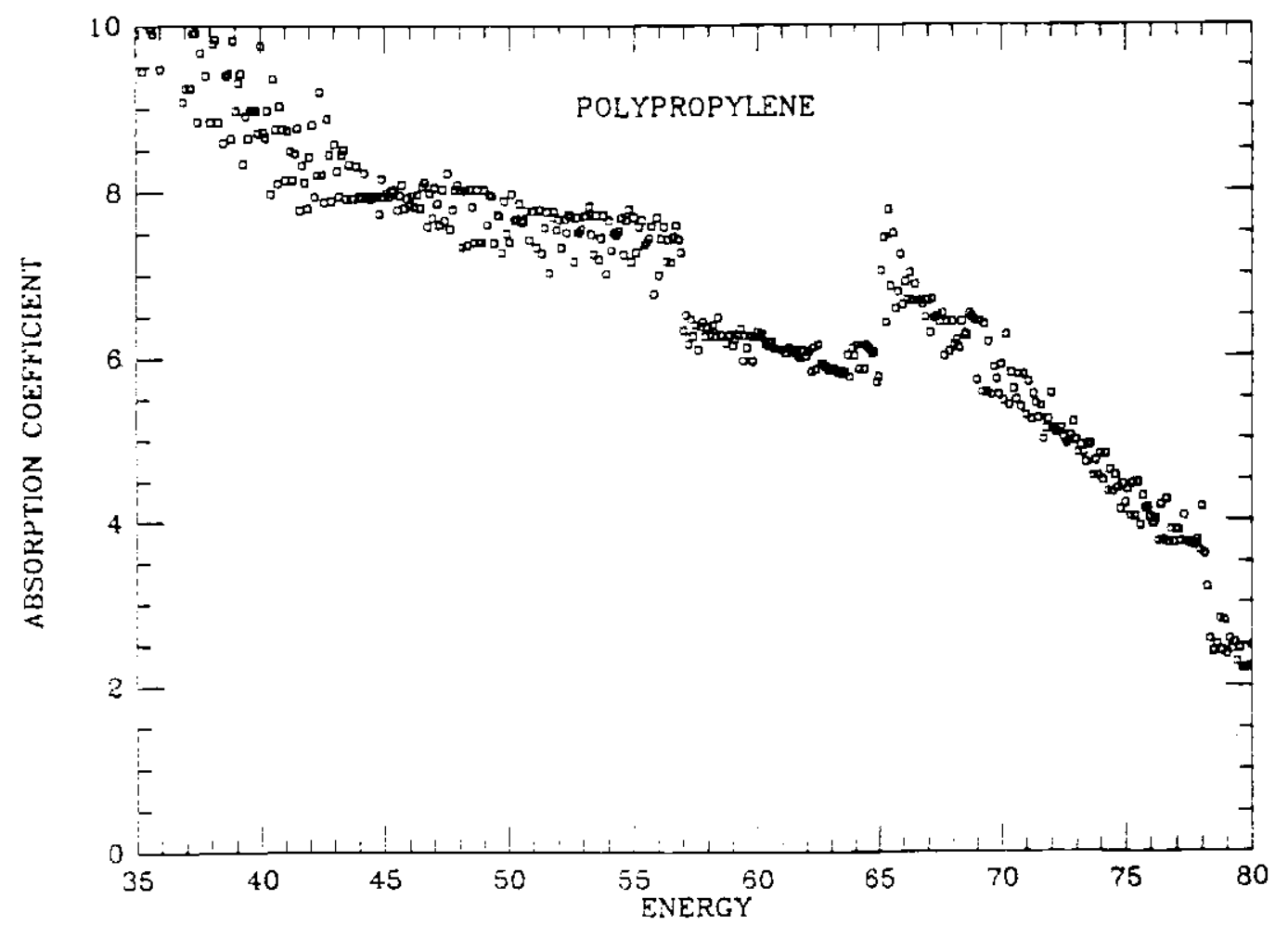

Figure 5: The mass absorption coefficient of polypropylene plotted with respect to energy. 


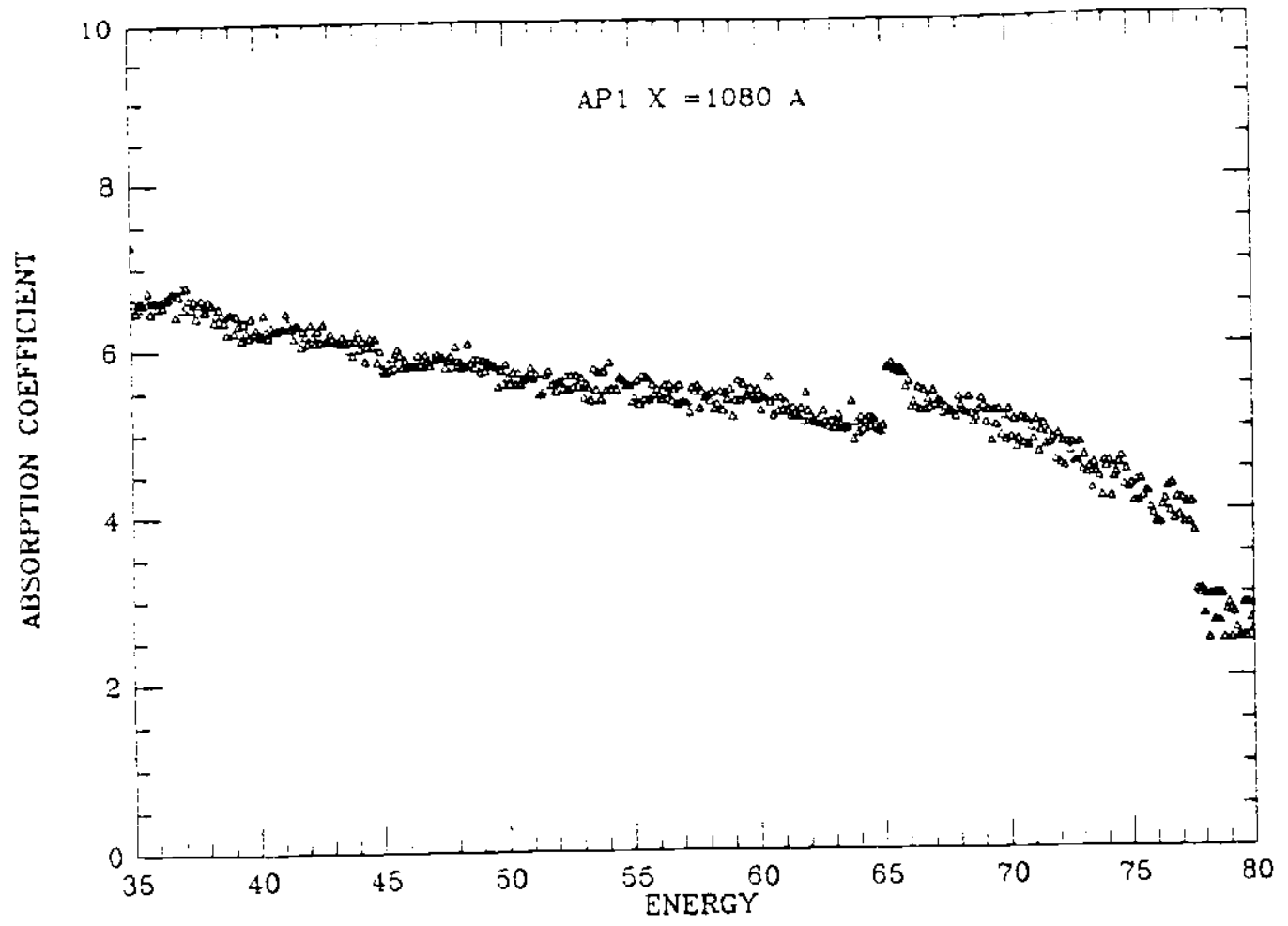

Figure 6: The mass absorption coefficient of AP-1 plotted with respect to energy.

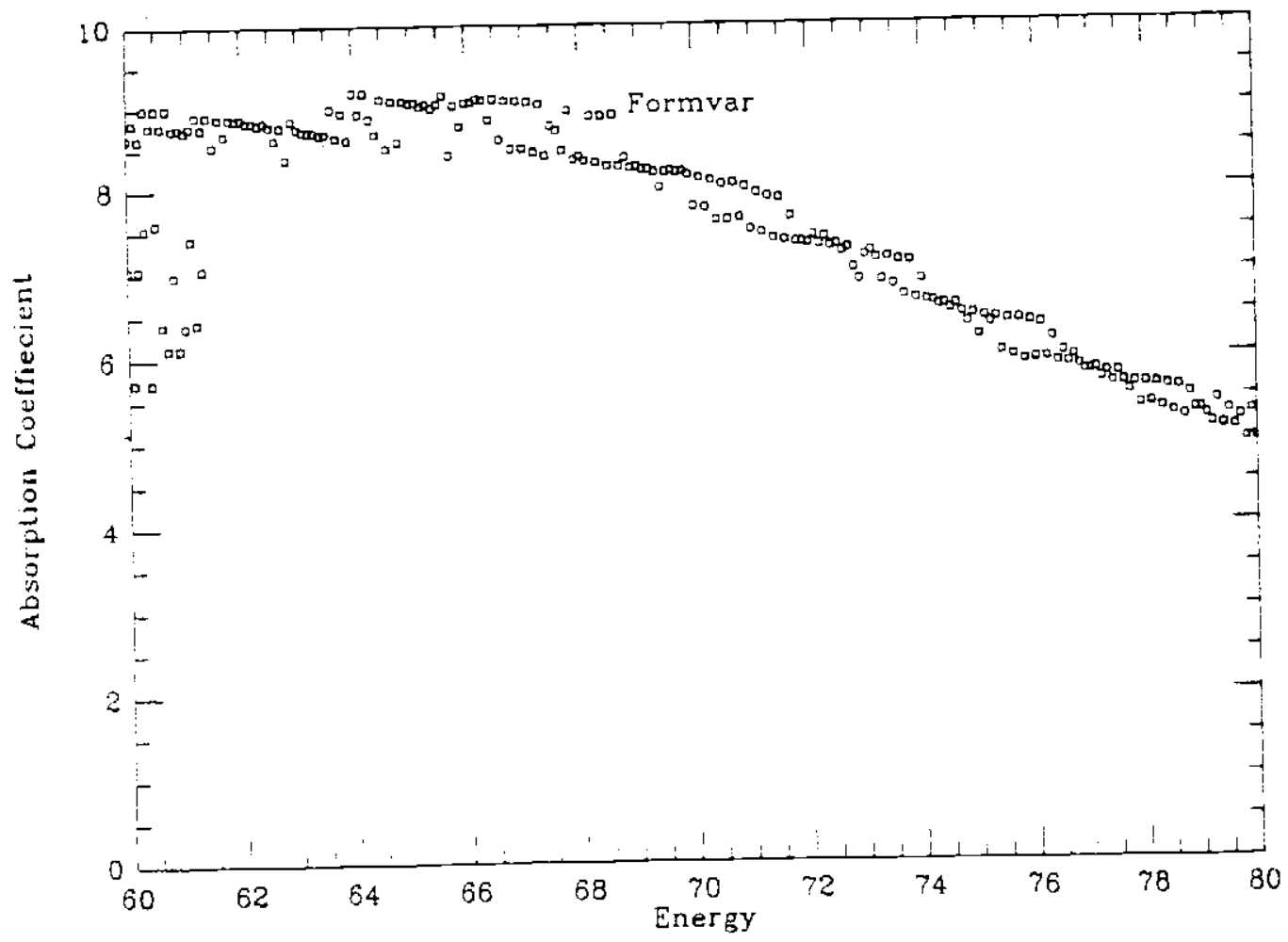

Figure 7: The mass absorption coefficient of Formvar plotted with respect to energy. 
disconcerting and indicate that the transmission values are accurate to only approximately $10 \%$.

Since the focus of our research is finding a filter with a passband at $72.5 \mathrm{eV}$, the remainder of the data is presented in a narrow band surrounding the area of interest. Figures 3 and 4 show the fractional transmission of AP1 and Formvar respectively. Although the theoretical predictions of the Formvar and polypropylene's transmission both match theory extremely well, the prediction for AP1 and the measured transmission differ by an average of about $30 \%$. Figures 5, 6 and 7 show the absorption coefficients of the materials with respect to energy. If all other factors were equal the material with the lowest absorption constant at $72.5 \mathrm{eV}$ would be preferred. Based upon the measurements made during the course of this experiment, that material would be AP1. This material is also preferred over the others investigated by reason of its ease in fabrication and mechanical toughness.

\section{CONCLUSIONS}

The synchrotron is a very useful radiation source for filter transmission measurements. The transmission of three polymers potentially useful for use as a supportive substrate for soft $\mathrm{x}$-ray filters were measured at over 1700 different energies between $10 \mathrm{eV}$ and $180 \mathrm{eV}$. Based upon these preliminary results, we favor AP-1 as the filter substrate material.

\section{ACKNOWLEDGEMENTS}

The authors would like to extend their sincere thanks to all of the BYU faculty who assisted in the completion of this paper: Drs. Douglas Jones, Ray Perkins, and the rest of the MOXTEK personnel. A special thanks is deserved by Professor Norman Tolk of Vanderbuilt University, who granted the use of his beamline for this experiment, and by his graduate students Dengfa Liu and David McClure, who contributed a significant amount of their time and knowledge in modifying the experimental chamber for our use.

\section{REFERENCES}

1. W.R. Hunter, "The Preparation and Use of Unbacked Metal Films as Filters in the Extreme Ultraviolet", Physics of Thin Films, vol. 7 p103 (1973).

2. MOXTEK Multilayer Optical and X-Ray Technology Inc., P.O. Box 7070 University Station, Provo, UT 84602.

3. E. Spiller, K. Grebe, and L. Golub, "Filters for Soft X-ray Solar Telescopes", SPIE vol. 1160 p66 (1989).

4. M.A. Spivack, "Parylene Thin Films for Radiation Applications" Review of Sci. Instr. vol. 41 No. 11 p1614 Nov. (1970).

5. B.L. Henke, "Some Notes on Ultrasoft X-ray Fluorescence Analysis- $10 \mathrm{~A}$ to $100 \mathrm{~A}$ Region", Adv. in X-ray Analysis, vol 8 p269 (1964). 
6. B.L. Henke, P. Lee, T.J. Tanaka, R. L. Shimabukuro, and B.K. Fujikawa, "Low-energy $\mathrm{X}$-ray interaction coefficients: photoabsorption, scattering, and reflection," At. Data Nucl. Data Tables 27, 1-144(1982) (and by the same authors) "The atomic scattering factor, f1 + if2, for 94 elements and for the 100 to $2000 \mathrm{eV}$ photon energy region," in AIP Conf. Proc. No. 75: Low Energy X-Ray Diagnostics, D.t. Attwood and B. L. Henke, eds., (1981) 